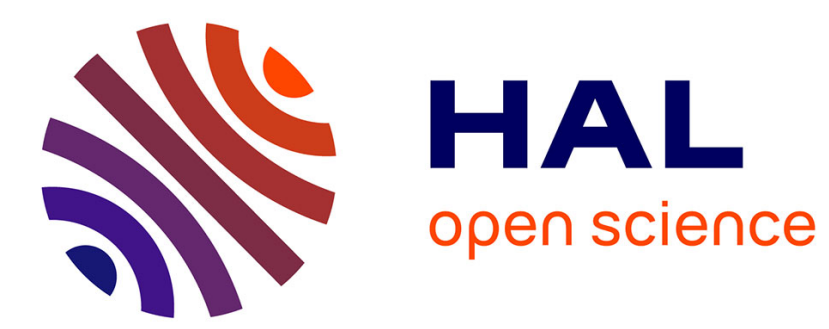

\title{
On a characterization of variance and covariance
}

Norbert Poschadel

\section{To cite this version:}

Norbert Poschadel. On a characterization of variance and covariance. Statistics and Probability Letters, 2010, 10.1016/j.spl.2010.07.018 . hal-00691787

\section{HAL Id: hal-00691787 \\ https://hal.science/hal-00691787}

Submitted on 27 Apr 2012

HAL is a multi-disciplinary open access archive for the deposit and dissemination of scientific research documents, whether they are published or not. The documents may come from teaching and research institutions in France or abroad, or from public or private research centers.
L'archive ouverte pluridisciplinaire HAL, est destinée au dépôt et à la diffusion de documents scientifiques de niveau recherche, publiés ou non, émanant des établissements d'enseignement et de recherche français ou étrangers, des laboratoires publics ou privés. 


\section{Accepted Manuscript}

On a characterization of variance and covariance

Norbert Poschadel

PII:

S0167-7152(10)00212-9

DOI:

10.1016/j.spl.2010.07.018

Reference: $\quad$ STAPRO 5756

To appear in: Statistics and Probability Letters

Received date: 14 January 2010

Revised date: 26 July 2010

Accepted date: 26 July 2010

Please cite this article as: Poschadel, N., On a characterization of variance and covariance.

Statistics and Probability Letters (2010), doi:10.1016/j.spl.2010.07.018

This is a PDF file of an unedited manuscript that has been accepted for publication. As a service to our customers we are providing this early version of the manuscript. The manuscript will undergo copyediting, typesetting, and review of the resulting proof before it is published in its final form. Please note that during the production process errors may be discovered which could affect the content, and all legal disclaimers that apply to the journal pertain. 


\title{
On a characterization of variance and covariance
}

\author{
Norbert Poschadel \\ Leibniz Institute for Farm Animal Biology (FBN), Genetics and Biometry, Wilhelm-Stahl-Allee 2 \\ D-18196 Dummerstorf, Germany
}

\begin{abstract}
Variance and standard deviation play a central role in probability and statistics. One reason for this might be that the variance of the sum of independent (even of uncorrelated) square integrable random variables is the sum of their variances. A generalization of this is the additivity of the covariance matrix for independent random vectors. We show that some kind of converse is also true: If for a "dispersion measure" $V$ of the form

$$
V(X)=E(f(X-E X)), \quad \text { where } f: \mathbb{R}^{n} \rightarrow \mathbb{R} \text { is even, i.e., } f(-x)=f(x) \forall x \in \mathbb{R}^{n},
$$

additivity $V(X+Y)=V(X)+V(Y)$ holds for every two independent $\mathbb{R}^{n}$-valued random variables $X$ and $Y$ (such that all involved integrals exist), then necessarily $f(x)=x^{\prime} A x$ for some symmetric $n \times n$-matrix $A$, and so $V$ is a linear combination of the covariances between any two components of $X$. For $n=1$ it follows that $V$ is a multiple of the variance and thus "variance" is not only a popular example for a dispersion measure with additivity for independent random variables, but it can even be characterized by this property.
\end{abstract}

\section{Statement of the problem}

Let $(\Omega, \mathcal{F}, P)$ be a probability space, $n \in \mathbb{N}$ and $X: \Omega \rightarrow \mathbb{R}^{n}$ a (coordinate-wise) integrable random variable. Henceforth the term "random variable" will be abbreviated with r.v. (or r.vs. for its plural form). The expectation $\int X d P \in \mathbb{R}^{n}$ and the distribution law of $X$ will be denoted by $E X$ and $P_{X}$, respectively. We are interested in studying values or parameters which are used to quantify the deviation of $X$ from $E X$.

While location parameters describe the "center" of a r.v., dispersion parameters are used to quantify its fluctuation. We think of a fixed $n$ and compile some notions (based on [8]) relevant to our analyses:

$A$ statistical parameter $S$ is an $\mathbb{R} \cup\{\infty\}$ - or $\mathbb{R} \cup\{-\infty\}$-valued functional on some class $\mathcal{C}$ of $\mathbb{R}^{n}$-valued r.vs. which depends only on the distribution $P_{X}$ of $X$, i.e., $S(X)=S(Y)$ if $P_{X}=P_{Y}$. We assume that $\mathcal{C}$ contains $X+Y$ whenever $X, Y \in \mathcal{C}$ are independent (implicitly presuming they are defined on the same probability space) and all constant r.vs., which will be briefly denoted by its values $d \in \mathbb{R}^{n}$. $S$ is called

- $a$ dispersion parameter, if for all $X \in \mathcal{C}$ and $d \in \mathbb{R}^{n}: S(X+d)=S(X)$.

- scale-equivariant, if for all $X \in \mathcal{C}$ and $\lambda \in[0, \infty[: \lambda X \in \mathcal{C}$ and $S(\lambda X)=\lambda S(X)$.

- independently additive, if $S(X+Y)=S(X)+S(Y)$ for all independent $X, Y \in \mathcal{C}$.

We want to use the location parameter $E X$ and want to measure deviations from, or fluctuations around $E X$ with dispersion parameters of the form

$$
V_{f}(X)=E(f(X-E X))
$$


where $f: \mathbb{R}^{n} \rightarrow \mathbb{R}$ is an even function, i.e., $f(-x)=f(x)$ for all $x \in \mathbb{R}^{n}$. Of special interest is the case $n=1$. Important examples in the one-dimensional case are the centered absolute $p$ th moments, which are given by

$$
V_{p}(X)=E\left(|X-E X|^{p}\right)
$$

for any $p>0$; the most important of them is the variance var $:=V_{2}$.

The variance (together with its square root, the standard deviation) is the most commonly used dispersion parameter for r.vs. Our aim is to understand the reasons for this. Already Gauss acknowledged in [3] that taking the square of the "error" (i.e. the deviation from the mean) is quite arbitrary, but he argued that it is very natural to do so, since squaring is doubtlessly the simplest (non constant) function which assigns a positive number to any (non null) error. However, this argument is more a heuristic one, obtained from practical considerations and not motivated by some "deeper insight".

A simple but often used property is

$$
\operatorname{var}(X+Y)=\operatorname{var}(X)+\operatorname{var}(Y)
$$

for all uncorrelated, especially for all independent, square integrable r.vs. $X$ and $Y$. We want to investigate the question whether the variance is characterized by independent additivity within the class of the functionals $V_{f}$. As an illustration we show first that the variance can be characterized by this property within the smaller class of all $V_{p}$. Let $X$ and $Y$ be independent and standard normally distributed real-valued r.vs. Both have the density

$$
\varphi(x)=\frac{1}{\sqrt{2 \pi}} e^{-\frac{x^{2}}{2}}
$$

and so

$$
V_{p}(X)+V_{p}(Y)=2 V_{p}(X)=2 \cdot \frac{1}{\sqrt{2 \pi}} \int_{-\infty}^{\infty}|x|^{p} e^{-\frac{x^{2}}{2}} d x .
$$

On the other hand, $X+Y$ has the density

$$
\varphi_{2}(x)=\frac{1}{\sqrt{2 \pi} \cdot \sqrt{2}} e^{-\frac{x^{2}}{2 \cdot 2}}
$$

and so

$$
V_{p}(X+Y)=\frac{1}{\sqrt{2 \pi} \cdot \sqrt{2}} \int_{-\infty}^{\infty}|z|^{p} e^{-\frac{z^{2}}{2 \cdot 2}} d z=\frac{1}{\sqrt{2 \pi}} \int_{-\infty}^{\infty}(\sqrt{2})^{p}|x|^{p} e^{-\frac{x^{2}}{2}} d x .
$$

Thus, $V_{p}(X)+V_{p}(Y)$ and $V_{p}(X+Y)$ coincide only if $2=(\sqrt{2})^{p}$, i.e. $p=2$.

One of our results will be that the variance is also characterized by independent additivity within the much larger class of the statistical parameters $V_{f}$, where $f: \mathbb{R} \rightarrow \mathbb{R}$ is an even measurable function. Note, however, that there are also situations where independent additivity is not of uttermost interest and/or other dispersion parameters can be better adapted or more useful. This is especially the case, if a dispersion parameter is required which is robust with respect to outliers, then the mean absolute deviation $V_{1}$ or some difference of quantiles can be a better choice.

To investigate the equation $V_{f}(X+Y)=V_{f}(X)+V_{f}(Y)$ we can, due to (1), assume without loss of generality that $E X=E Y=0$. It is known that the variance is not characterized by independent additivity within the larger class of all functionals $V_{f}$ of the form (1) when $f$ is not required to be even: For the 3rd centralized moment $T$, given by $T(X)=E\left((X-E X)^{3}\right)$, we have $T(X+Y)=T(X)+T(Y)$ for all independent r.vs. $X$ and $Y$ with integrable 3 rd power; to see this, assume w.l.o.g. $E X=E Y=0$ and take the expectation over the equation $(X+Y)^{3}=X^{3}+3 X^{2} Y+3 X Y^{2}+Y^{3}$. 
Returning to the multivariate case, with an arbitrary $n$, we may ask for which measurable even functions $f: \mathbb{R}^{n} \rightarrow \mathbb{R}$ the statistical parameter $V_{f}$ given by (1) is independently additive, i.e. it holds $V_{f}(X+Y)=V_{f}(X)+V_{f}(Y)$ for independent $\mathbb{R}^{n}$-valued r.vs. $X$ and $Y$.

One might be interested in further generalizing the question of independent additivity and ask for vectoror matrix-valued statistical parameters $S: \mathcal{C} \rightarrow \mathbb{R}^{k}$ or $S: \mathcal{C} \rightarrow \mathbb{R}^{k \times l}$ with $S(X+Y)=S(X)+S(Y)$ for independent $X$ and $Y$, where $\mathcal{C}$ is (as before) some class of $\mathbb{R}^{n}$-valued r.vs. A well-known and natural example of this type is provided by the covariance matrices

$$
\operatorname{Cov}(X):=\left(\operatorname{cov}\left(X_{i}, X_{j}\right)\right)_{i, j=1 \ldots, n}=\left(E\left[\left(X_{i}-E X_{i}\right)\left(X_{j}-E X_{j}\right)\right]\right)_{i, j=1, \ldots, n}
$$

for coordinate-wise square integrable $\mathbb{R}^{n}$-valued r.vs. $X=\left(X_{1}, \ldots, X_{n}\right)^{\prime}$ (here $x^{\prime}$ denotes the transpose of a vector $x$ ). However, such a "statistical parameter" $S$ is independently additive if and only if each of its components (coordinates or matrix elements) has this property. So there is no need to consider such "higher dimension-valued statistical parameters" $S$ and, thus, we won't do this.

\section{The main theorem}

According to the last example in Section 1, the covariance $\operatorname{cov}\left(X_{i}, X_{j}\right)$ between any two fixed components $X_{i}$ and $X_{j}$ of square integrable $n$-dimensional r.vs. $X=\left(X_{1}, \ldots, X_{n}\right)^{\prime}$ is an independently additive statistical parameter. So any linear combination of them is also of this kind. In this section we will show that, conversely, every independently additive statistical parameter of the form (1) is such a linear combination.

Let us call an $\mathbb{R}^{n}$-valued r.v. $X$ a symmetric balance, if it attains only the values $a \in \mathbb{R}^{n}$ and $-a$, each with probability $\frac{1}{2}$ (or with probability 1 , if $a=0$ ), i.e. we have

$$
P_{X}=\beta_{a}:=\frac{1}{2} \delta_{a}+\frac{1}{2} \delta_{-a},
$$

where $\delta_{x}$ denotes the Dirac measure concentrated at some point $x \in \mathbb{R}^{n}$. Using balances we can recover an even function $f$ from $V_{f}$ via $f(a)=V_{f}(X)$ for $X$ with $P_{X}=\beta_{a}$.

Theorem 2.1. Let $n \in \mathbb{N}$ and $f: \mathbb{R}^{n} \rightarrow \mathbb{R}$ be an even Borel-measurable function. Let $V_{f}(X)$ be given by (1) for any integrable $\mathbb{R}^{n}$-valued r.v. $X$ for which this integral exists. If

$$
V_{f}(X+Y)=V_{f}(X)+V_{f}(Y)
$$

for (at least) all independent symmetric balances $X$ and $Y$, then there exists a symmetric real $n \times n$-matrix $A$ such that $f(x)=x^{\prime} A x$ for all $x \in \mathbb{R}^{n}$ and thus

$$
V_{f}(X)=E\left((X-E X)^{\prime} A(X-E X)\right)
$$

for all $X$ in the domain of definition of $V_{f}$.

Proof. Choosing for $a$ and $b \in \mathbb{R}^{n}$ independent symmetric balances $X$ and $Y$ with distributions $P_{X}=\beta_{a}$ and $P_{Y}=\beta_{b}$, independent additivity (4) leads to

$$
\frac{1}{4}(f(a+b)+f(a-b)+f(-a+b)+f(-a-b))=V_{f}(X+Y)=V_{f}(X)+V_{f}(Y)=f(a)+f(b) .
$$

Due to $f(-x)=f(x)$ this is equivalent to the the parallelogram equation

$$
f(a+b)+f(a-b)=2 f(a)+2 f(b) \quad\left(a, b \in \mathbb{R}^{n}\right) .
$$


Choosing $a=b=0$ yields $f(0)=0$ and choosing $b=a$ (for arbitrary $a$ ) then yields $f(2 a)=4 f(a)$ for all $a \in \mathbb{R}^{n}$. It is well-known from functional analysis that

$$
h(x, y)=\frac{1}{4}(f(x+y)-f(x-y)) \quad\left(x, y \in \mathbb{R}^{n}\right)
$$

defines a symmetric biadditive form $h$ on $\mathbb{R}^{n} \times \mathbb{R}^{n}$, i.e., $h(y, x)=h(x, y)$ and $h(x+y, z)=h(x, z)+h(y, z)$ for all $x, y, z \in \mathbb{R}^{n}$ (cf. [4], Lema 1, p. 16 or [1], Proposition 1 in 11.1). Further, $f(x)=h(x, x)$ for all $x$. Biadditivity leads to

$$
h(\lambda x, y)=h(x, \lambda y)=\lambda h(x, y) \quad \forall x, y \in \mathbb{R}^{n} \quad \text { and } \quad \lambda \in \mathbb{Q} .
$$

If $f$ is continuous then also $h$ is continuous and the last equation is valid for all $\lambda \in \mathbb{R}$. In this case $h$ is a symmetric bilinear form and so there exists a symmetric $n \times n$-matrix $A$ such that $h(x, y)=x^{\prime} A y$ for all $x, y \in \mathbb{R}^{n}$, especially

$$
f(x)=h(x, x)=x^{\prime} A x \quad \forall x \in \mathbb{R}^{n} .
$$

This proves the theorem for continuous $f$. To treat the general case we have to show that in this case, too, $h$ is bilinear and the same proof applies. We will show the homogeneity of $h$ without loss of generality in the first variable, i.e., $h(\lambda x, y)=\lambda h(x, y)$ for all $\lambda \in \mathbb{R}$. To this end, define for fixed $x, y \in \mathbb{R}^{n}$ the function $\ell_{x, y}: \mathbb{R} \mapsto \mathbb{R}$ by $\ell_{x, y}(\lambda):=h(\lambda x, y)$. Then $\ell_{x, y}$ is additive because of the additivity of $h$ in the first variable, i.e.,

$$
\ell_{x, y}(\lambda+\mu)=\ell_{x, y}(\lambda)+\ell_{x, y}(\mu) \quad \forall \lambda, \mu \in \mathbb{R} .
$$

This is Cauchy's functional equation. $h$ is (together with $f$ ) Borel-measurable and so $\ell_{x, y}$ is also Borelmeasurable. Consequently, there exists some $c \in \mathbb{R}$, possibly depending on $x$ and $y$, such that $\ell_{x, y}(\lambda)=c \lambda$ $\forall \lambda \in \mathbb{R}$ (this is a well-known property of measurable solutions of Cauchy's equation, see e.g. [2], [11] or [9]), that is

$$
h(\lambda x, y)=\ell_{x, y}(\lambda)=c \lambda \quad \forall \lambda \in \mathbb{R} .
$$

Choosing $\lambda=1$ yields $c=h(x, y)$ and thus our goal: $h(\lambda x, y)=c \lambda=\lambda h(x, y) \forall \lambda \in \mathbb{R}$.

\section{Characterizations of the variance by independent additivity}

This section is devoted to the one-dimensional case. First we obtain a characterization of the variance by independent additivity as a corollary of Theorem 2.1 and compare it with some related results in the literature. Then we take a closer look at scale-equivariant statistical parameters.

Corollary 3.1. Let $f:\left[0, \infty\left[\rightarrow \mathbb{R}\right.\right.$ be a Borel-measurable function. Let $V_{f}(X)=E(f(|X-E X|))$ for any integrable real-valued r.v. $X$ for which this integral exists. If $V_{f}(X+Y)=V_{f}(X)+V_{f}(Y)$ for (at least) all independent real-valued symmetric balances $X$ and $Y$, then there is a constant $A \in \mathbb{R}$, such that $f(x)=A \cdot x^{2}$ for all $x \geq 0$, and so $V_{f}$ is (null or) equal to $A \cdot$ var.

Proof. Set $n=1$ and let the even function $\mathbb{R} \ni x \mapsto f(|x|)$ take the place of the $f$ in Theorem 2.1.

We compare this result with some related work:

(a) In [6], 1.14, was given a similar characterization of the variance: The set $\operatorname{Prob}_{\infty}(\mathbb{R})$ of all probability measures on the Borel $\sigma$-Algebra of $\mathbb{R}$ with existing moments of any order is equipped with the convolution $*$. A function $\kappa: \operatorname{Prob}_{\infty}(\mathbb{R}) \rightarrow \mathbb{R}$ is called additive, if $\kappa(P * Q)=\kappa(P)+\kappa(Q)$ for all $P, Q \in \operatorname{Prob}_{\infty}(\mathbb{R})$. According to Theorem 1.11 of [6] a function $\kappa: \operatorname{Prob}_{\infty}(\mathbb{R}) \rightarrow \mathbb{R}$ is "continuous" and additive iff it is a 
(finite) linear combination of so-called "cumulants" $\kappa_{l}(l \in \mathbb{N})$; and according to 1.14 in [6] it is, in addition, nonnegative, iff it is a nonnegative multiple of the cumulant $\kappa_{2}$, which coincides with the variance. "Continuity" refers to a topology on $\operatorname{Prob}_{\infty}(\mathbb{R})$ explained in $[6]$ and the notion of "cumulant" was also provided there: $\kappa_{1}(P)$ is the expectation $\left(\int x P(d x)\right), \kappa_{2}(P)$ the variance and $\kappa_{3}(P)$ the 3 rd centralized moment of $P \in \operatorname{Prob}_{\infty}(\mathbb{R})$ (the other cumulants, too, are in a certain relation to the moments of $P$ ). In contrast to our Corollary 3.1, the characterization 1.14 in [6]

- needs strong regularity assumptions (existence of all moments, continuity),

- was proved using quite sophisticated techniques,

- but does not presume a special form like our $V_{f}$ in (1) for the additive functions considered.

So these two characterizations complement each other in some way.

(b) In [5] variance is characterized as the only nonnegative dispersion parameter $S$ on the class $\mathcal{C}_{\text {fin }}$ of the r.vs. which attain only finitely many real values, such that $S(X)=1$ if $P_{X}=\beta_{1}$ (a normalization), some continuity property holds, and

$$
S\left(\sum_{i=1}^{m} X_{i}\right)=\sum_{i=1}^{m} S\left(X_{i}\right)
$$

for any number $m$ of pairwise independent $X_{i} \in \mathcal{C}_{\text {fin }}$. As outlined in [5] and [6], the last condition is rather confining and seems not to be suitable as an axiom for deviation measures.

(c) In [7] the quite general functional equation

$$
E(f(\psi(X, Y)))=T(E(f(X)), E(f(Y)))
$$

for independent real-valued r.vs. $X$ and $Y$ is investigated ( $\psi$ and $T$ are to be determined, [10] treats the case $\psi(x, y)=x+y$, and $\psi(x, y)=T(x, y)=x+y$ corresponds to our situation). So they only consider statistical parameters $S_{f}(X):=E(f(X))$, rather than our $V_{f}$ 's. However, $V_{f}$ cannot be expressed as any $S_{g}$ unless $V_{f}$ is constant. Thus, [7] and [10] only investigate statistical parameters which lie outside the class of the functionals we are interested in.

Finally, we want to shed some light on the role played by scale-equivariance for dispersion parameters. There are dispersion parameters which are not of the form (1), some important of them are the $\sigma_{p}$ for $1 \neq p>0$, given by $\sigma_{p}:=\left(V_{p}\right)^{1 / p}$. The motivation for introducing them is that they are scale-equivariant, which seems to be a very desirable property for dispersion parameters. However, it is in contradiction with independent additivity. $V_{f}$ is equal to $A$ - var by Corollary 3.1 and thus not scale-equivariant (for $A \neq 0$ ). But it might still be conceivable that there is some other dispersion parameter $V$, not of the form (1), which is independently additive and scale-equivariant. We show that the existence of such a $V$ can also be excluded under some very mild conditions: Let $X$ and $Y$ be independent r.vs., both with density $\varphi$ given by (2). So $\sqrt{2} \cdot X$ and $X+Y$ are equally distributed with the density $\varphi_{2}$ given by (3). Let us assume that there is some statistical (not necessarily dispersion-) parameter $V$ which is independently additive and scale-equivariant. We further assume that $V$ is defined for the r.vs. with densities $\varphi$ or $\varphi_{2}$ and that it assigns finite values $\neq 0$ to them. Then

$$
\sqrt{2} \cdot V(X)=V(\sqrt{2} \cdot X)=V(X+Y)=V(X)+V(Y)=2 \cdot V(X),
$$

which is a contradiction, since $V(X)$ is neither $\pm \infty$ nor 0 . We sum up these observations:

\section{There is no "sensible" scale-equivariant independently additive dispersion parameter!}

The generalization to the multivariate case is straightforward. 


\section{Acknowledgement}

I am indebted to an unknown referee for valuable hints and questions, which led to a considerable improvement of this work.

\section{References}

[1] J. Aczél and J. Dhombres. Functional equations in several variables. Cambridge University Press, 1991.

[2] S. Banach. Sur l'équation fonctionnelle $\mathrm{f}(\mathrm{x}+\mathrm{y})=\mathrm{f}(\mathrm{x})+\mathrm{f}(\mathrm{y})$. Fundam. Math., 1:123-124, 1920.

[3] C.F. Gauss. Méthode des Moindres Carrés. Mémoire sur la Combination des Observations. Transl. J. Bertrand, Mallet-Bachelier, Paris, 1855.

[4] S. Kurepa. Funkcionalna Analiza. Školska Knjiga, Zagreb, 1990.

[5] M. Martin Diaz. Caracterización de la varianza. Trab. Estad. Invest. Oper., 28:85-97, 1977.

[6] L. Mattner. What Are Cumulants ? Doc. Math. J. DMV, pages 601-622, 1999.

[7] P. Muliere. Una nota su operazioni associative, transformate integrati e problemi di caratterizzazione in statistica. Rivista di matematica per le scienze economiche e sociali - Anno $\%$. Fascicoli $1^{\circ}-2^{\circ}$, pages 79-93, 1984.

[8] G.C. Pflug and W. Römisch. Modeling, measuring and managing risk. World Scientific Publishing Co. Pte. Ltd., New Jersey, London, Singapore, 2007.

[9] N. Poschadel. Über die abstandsverträglichen Abbildungen auf dem Kreis und auf der reellen Geraden. Math. Semesterber., 49:45-54, 2002.

[10] L.J. Savage. The characteristic function characterized and the momentousness of moments. In: Studi di probabilità, statistica e ricerca operativa in onore di Giuseppe Pompilj, pages 131-141. Tipografia Oderisi Editrice, Gubbio, 1971. Reprinted in: The writings of Leonard Jimmie Savage : A memorial selection, pages 615-625. The American Statistical Association and The Institute of Mathematical Statistics, 1981.

[11] W. Sierpiński. Sur l'équation fonctionnelle $\mathrm{f}(\mathrm{x}+\mathrm{y})=\mathrm{f}(\mathrm{x})+\mathrm{f}(\mathrm{y})$. Fundam. Math., 1:116-122, 1920. 